\title{
List of Reviewers 2017
}

The Journal of Pediatric Rehabilitation Medicine would like to recognize the following reviewers for their time and commitment in providing scientific peer reviews in the past year.

\author{
Rishi Agrawal \\ Unoma Akamagwuna \\ Lusine Ambartsumyan \\ Antonio Andreacchio \\ Juan Carlos Arango-Lasprilla \\ Ian Baguley \\ Laurence Baskin \\ Rolf Beetz \\ Patricia Beierwaltes \\ Patrizia Bertolini \\ Rhonda Bolin \\ Elisabet Bousquet \\ Robin Bowman \\ Timothy Brei \\ Cathleen Buckon \\ Wendy Burdo-Hartman \\ Robert Burke \\ Kristen Campbell \\ Federico Canavese \\ Arturo Carta \\ Enrico Castelli \\ Jonathan Castillo \\ Rajeev Choudhry \\ David Chu \\ Paige Church \\ Jerry Clayton \\ Douglass Clayton \\ Robert Cohn \\ Patty Coker-Bolt \\ Robin Cotton \\ Bethany Coyne \\ Theresa Crytzer \\ Giovanni Cucchiaro \\ Anna Cuomo \\ Shay Dawson \\ Mark Dias \\ Brad Dicianno \\ Margie DiCuccio \\ Marcel Dijkers
}

\author{
Cynthia Dodds \\ Nancy Dodge \\ Nienke Dosa \\ Anne Dudley \\ Rochelle Dy \\ Jonathan Ellison \\ Raul Escobar \\ Maya Evans \\ Deborah Fels \\ Jack Fletcher \\ Edward Fong \\ Ellen Fremion \\ Michael Green \\ Toyia Greene \\ Scott Grosse \\ Juliet Haarbauer-Krupa \\ John Hanks \\ Heather Hardin \\ Solveig Hart \\ Caroline Hastings \\ Lisa Herzig \\ Gregory Heuer \\ Grayson Holmbeck \\ Betsy Hopson \\ Katherine James \\ Kristin Jensen \\ Abigail Johnson \\ David Joseph \\ Denise Justice \\ Abdol-Mohammad Kajbafzadeh \\ Vijaya Kancherla \\ Sandra Kaplan \\ Jerie Karkos \\ Elana Katz \\ Maryellen Kelly \\ Virginia Kharasch \\ Sunkyung Kim \\ Shelly King \\ Shyam Kishan
}


L. Andrew Koman

Dennis Kuo

Brad Kurowski

Eberhard Kuwertz-Broeking

Charlotte Leboeuf-Yde

Seung-Won Lee

Abigail Levin

Kaitlin Lewis

Yuan Li

Lynne Logan

Russell Mardon

Joyce Maring

Andrea Martinuzzi

Janette McDougall

Matthew McLaughlin

Renato Melo

Emilia Michou

Rosalie Misseri

Olga Morozova

Shubhra Mukherjee

Lizanne Mulligan

Nancy Murphy

Sarah Murphy

Emily Myers

Erin Naber

Virginia Nelson

Jacob Neufeld

Michael Nigro

Garey Noritz

Marykay Nutini

Joe O'Neil

Pornsuree Onmanee

Arve Opheim

Lijing Ouyang

Britt Pados

Dae-Sung Park

Michael Partington

Peter Patrick

Iris Perez

Paula Peterson

Moya Peterson

Elaine Pico

Frank Pidcock

David Pruitt

Alex Psihogios

Jennifer Queally

Sarah Reedman

Andrew Reisner

Mark Rich

Monique Ridosh
Andrew Ries

Robert Rinaldi

Elias Rizk

Jacob Robison

Brandon Rocque

Peter Rosenbaum

Nathan Rosenberg

Samuel Rosenfeld

Adam Rush

Kashika Sahay

Jose Salazar-Torres

Cynthia Salorio

Kathy Sawin

Veronica Schiariti

Lee Segal

Danielle Shapiro

Justine Joan Sheppard

David Sherry

Tamara Simon

Kathryn Smith

Virginia Stallings

Stacy Suskauer

Hubert Swana

Mary Swiggum

Konrad Szymanski

Stacey Tanaka

Ravi Thiagarajan

Judy Thibadeau

Pam Thomason

Rick Tivis

Tereen Toles

Raymond Tse

Margaret Turk

Alex Van Speybroeck

Kerri Vanderbom

Chantelle Varnado

William Walker, Jr. MD

M. Chad Wallis

Kate Wan-Chu Chang

Yinding Wang

Seth Warschausky

John Weiner

Jennifer Wilhelmy

Pam Wilson

Donna Woods

Gordon Worley

Joseph (Zhe) Xu

Nancy Yeh

Elizabeth Yerkes

Stephen Zderic 